CAD deemed to be clinically equipoised following HT discussion. These results suggest that PCI is non-inferior to CABG in this population and provide validation to the decision making process of the HT in real life.

Conflict of Interest nil

\section{AN UNUSUAL CAUSE OF DYSPNOEA DIAGNOSED} DURING RIGHT HEART CATHETERISATION

James O'Brien, Rahul Chattopadhyay, Peter J Pugh. Cambridge University Hospital, Cambridge, UK

\subsection{6/heartjnl-2021-BCS.63}

Background It is common for patients with exertional dyspnoea to be referred to Cardiology for investigation. This case highlights a rare but important cause of cyanosis, diagnosed after extensive investigations.

Case Presentation A 73-year-old was referred to the Respiratory Medicine team with limiting exertional dyspnoea. Initial standard investigations proved unremarkable, with normal spirometry, CT pulmonary angiogram, V/Q scan, 6-minute walk test and normal $\mathrm{PaO} 2$ on arterial blood gas sampling. The patient was then referred to Cardiology for consideration of a cardiac cause of her symptoms. Her past medical history included rheumatoid arthritis, retinal vein thrombosis and idiopathic right arm thrombosis. After her initial review by Cardiology, a decision was made to perform both a coronary angiogram and right heart catheterisation. The coronary arteries showed no significant flow limiting lesion and the pulmonary pressures were not significantly elevated with no evidence of a shunt During the procedure, it was noted that the patient's blood was dark brown in colour (figure 1), with low systemic (66\%) and pulmonary artery $(48.6 \%)$ oxygen saturation as measured on the cath lab oximeter, with normal respiratory rate (12/minute). Arterial blood was mixed in a syringe with $100 \%$ oxygen, which produced no change in the colour. A full blood gas analysis was therefore undertaken, which identified a disparity between the measured $\mathrm{PaO} 2(12.0 \mathrm{kPa})$ and oxygen saturations. A haemoglobinopathy was therefore suspected, which was felt most likely to be a case of methaemoglobinaemia. However, it proved not possible to measure this on the multiple point of care ABG machines, nor at the main biochemistry laboratory. The patient was subsequently investigated extensively by Haematology, ultimately identifying diagnosis of sulfhaemoglobinaemia. The source for the sulphated haemoglobin was due to an Epsom salt (MgSO4) containing tonic the patient was using for constipation. The patient was treated with exchange transfusions under the care of the haematology team alongside stopping the Epsom salt tonic.

Discussion Sulfhaemoglobinaemia (SulfHb) is a rare haemoglobinopathy which arises due to the sulphation of haemoglobin, this results in a significantly reduced oxygen carrying capacity due to reduced affinity for oxygen (Lu et al., 1998); it is often reported that the source of exogenous sulphur is from medications, bowel pathology or occupational exposures. This case highlights the importance of right heart catheterisation as an investigation to help differentiate the causes of exertional dyspnoea and the importance of considering full blood gas analysis when the numbers do not add up.

Conclusion Sulfhaemoglobinaemia is a rare cause of exertional dyspnoea and should be considered in patients with a clear

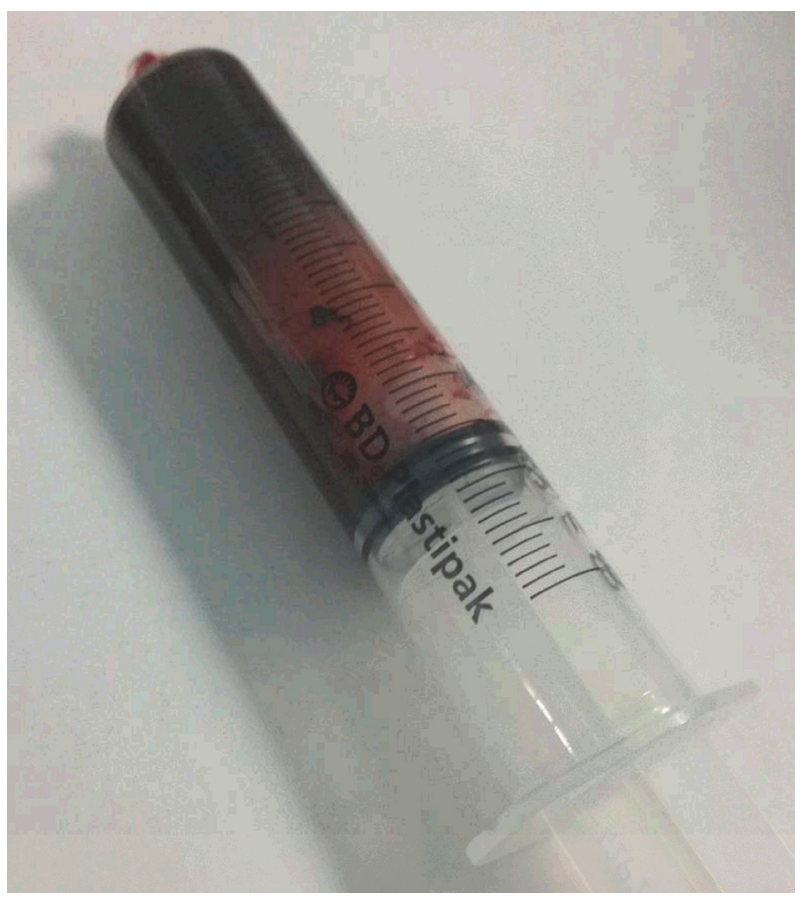

Abstract 63 Figure 1

disparity between saturations measured by pulse oximetry and atrial blood sampling.

Conflict of Interest None

\section{USE OF SIROLIMUS-COATED BALLOON IN DE NOVO LESIONS; MID-TERM FOLLOW-UP FROM A SINGLE- CENTRE REGISTRY}

Bhagya Harindi Loku Waduge, Harkaran Kalkat, Ameenathul Mazaya Fawzy, Abdullah Saif, Sampath Athukorala, Gurbir Bhatia, Nitin Kumar, Bethan Freestone, Jerome Ment Kaeng Lee, Michael Pitt, George Pulikal, Sandeep Basavarajaiah Sandeep Basavarajaiah. Birmingham Heartlands Hospital, Birmingham, UK

\subsection{6/heartjnl-2021-BCS.64}

Aims Use of drug coated balloon (DCB) in coronary intervention is escalating and the guidelines recommend its use in restenotic lesions. However, some of the recent data suggest, it can also be considered in subset of denovo lesions especially; small vessels, ostium of an important side-branch and in patients unable to tolerate dual antiplatelet therapy. Sirolimus is the drug of choice in currently available drug eluting stents. Most DCBs used elute Paclitaxcel, but there is no data on Sirolimus coated DCBs. In this study, we report outcomes from the use of a Sirolimus coated balloon (SCB) in de novo coronary lesions.

Methods We included all patients treated with an SCB between April and December 2018. Outcomes measured include cardiac death, target vessel myocardial infarction, target lesion revascularisation, target vessel revascularisation and MACE (combination of cardiac death, target vessel MI and TLR).

Results During the study period, 351 patients (with 414 lesions) with de novo lesions were treated with an SCB. The mean age of patients was $65.6 \pm 11.5$ years, 275 (78\%) were male and $39 \%(n=212)$ had diabetes. Most lesions treated were in the LAD/diagonal system $(n=173,42 \%)$. Predilatation 
was performed in 98\% (405 lesions). Bailout stenting (with a drug eluting stent) was required in $7 \%$ lesions $(n=30)$ and of which 11 were due to dissections and 19 were due to recoil $>50 \%$ following DCB use. The mean diameter and length of DCBs were $2.47 \mathrm{~mm}$ and $26 \mathrm{~mm}$ respectively. During a median follow-up of 322-days (11-months) cardiac death was reported in 8 patients (2.3\%). Target vessel MI was in 3\%; $\mathrm{n}=10$, TLR and TVR per lesion were $8 \%(\mathrm{n}=34)$ and $9 \%$ $(n=37)$ respectively. The MACE rate was $11 \%$. There were no documented cases of acute vessel closure.

Conclusion The mid-term outcome from the first ever study on sirolimus coated balloons in de novo lesions appears promising with low rates of hard endpoints with no documented case of acute vessel closure. The MACE rates appear to support the efficacy of SCBs in de novo lesions. However, we need longer follow-up which is on-going, and we will be able to report outcomes from even longer follow-up during the presentation.

Conflict of Interest None to declare

\section{LEFT MAIN STEM PERCUTANEOUS CORONARY INTERVENTION IN A NON-SURGICAL CENTRE - A SAFE AND EFFECTIVE TREATMENT OPTION}

Joanna Abramik, Sathish Parasuraman, Mohammad Sahebjalal, Thomas Burchell, Michael Seddon. Taunton and Somerset NHS Foundation Trust, Taunton, UK

\subsection{6/heartjnl-2021-BCS.65}

Introduction The ESC/EACTS 2018 guidelines on myocardial revascularisation supported the use of percutaneous coronary intervention (PCI) and coronary artery bypass grafting (CABG) for left main stem (LMS) disease of low complexity, and recommended CABG over PCI in disease of intermediate or high complexity. Following revelations of EXCEL study data, the guidance is being reviewed. Meanwhile, patients with LMS disease continue to present in both elective and acute settings, and clinicians face complex decision-making, which can be particularly challenging in non-surgical centres.

Methods The aim of this study was to investigate the safety and efficacy of LMS PCI in our non-surgical, 24/7 primary PCI centre. We have performed a retrospective analysis of 136 patients who underwent LMS PCI between 2015-2019 in both elective and acute (Non ST elevation-ACS and ST elevation MI) settings. The median follow up time was 19 months (range 12-59 months). Inpatient mortality and complication rates and all-cause mortality over the follow up period were assessed. We also examined use of the MDT and intracoronary imaging rates.

Results The number of PCI for LMS in both elective and acute settings steadily increased over the study period (figure 1). The patient and procedural characteristics are presented in tables 1 and 2, demonstrating appropriate patient selection (low risk anatomy, low percentage diabetes, predominant single stent strategy) and significantly increasing use of intracoronary imaging over time (figure 2). There was documented evidence of formal MDT discussion, or informal discussion with colleagues and the patient, in $82.1 \%$ of elective cases. In elective patients there were no in-hospital complications. In the acute cohort, we observed 2 post-procedural acute renal failure episodes, 1 embolic stroke and 1 episode of anaemia requiring blood transfusion. Overall, all-cause mortality at a median 19 months was $15.5 \%$ (21 of 136 patients). There were 4 deaths among elective patients and 17 in ACS patients. Higher death rate in the acute cohort was partly driven by cardiogenic shock in STEMI patients. 8 patients required further PCI for in-stent restenosis.

Conclusions In an all-comer population, including those with cardiogenic shock, LMS PCI seems to be effective with

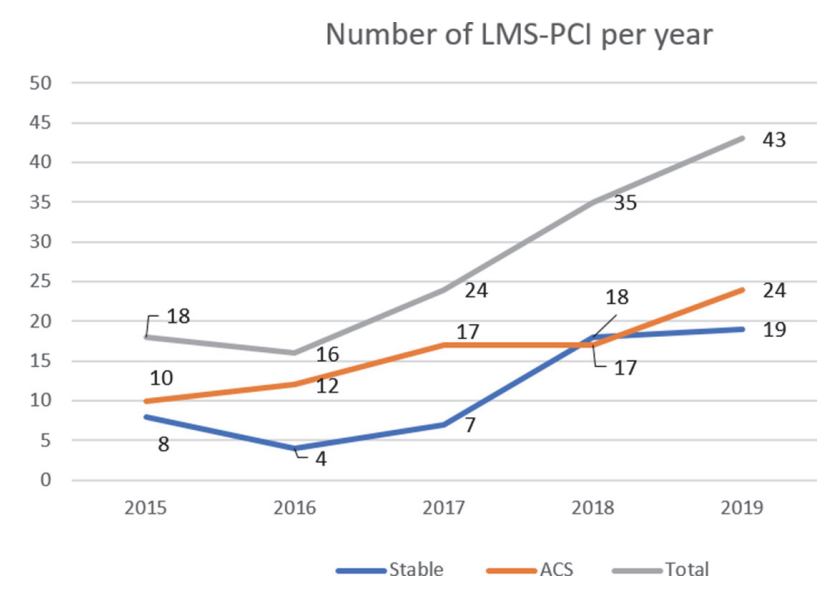

Abstract 65 Figure 1 Number of t MS-PCI performed between 20152019

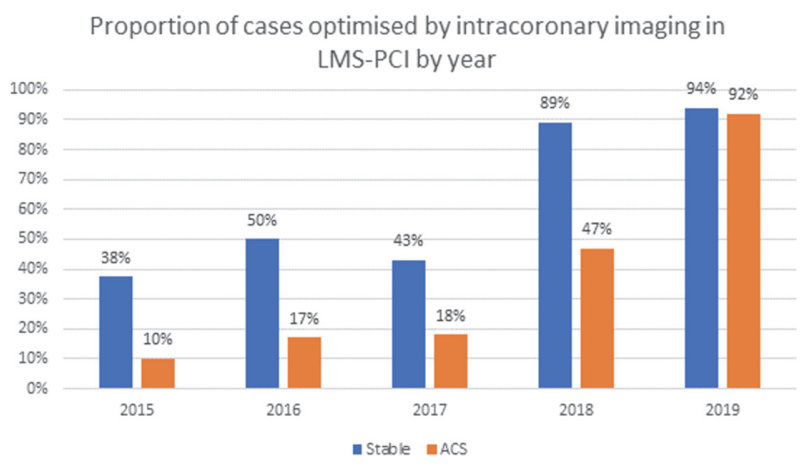

Abstract 65 Figure 2 Proportion of cases optimised by intracoronary imaging in LMS-PCI by year

Abstract 65 Table 1 Patient characteristics

\begin{tabular}{|l|l|l|}
\hline & Elective & Acute \\
\hline Number of patients, $n$ & 56 & 80 \\
\hline Age, median (range) & $71.5(53-87)$ & $70.5(22-91)$ \\
\hline Sex (male) $n,(\%)$ & $43(77.7)$ & $49(61.3)$ \\
\hline Diabetes, $n(\%)$ & $12(21.4)$ & $14(17.5)$ \\
\hline In-patient complications $n,(\%)$ & 0 & $4(5)$ \\
\hline In-patient mortality, $n(\%)$ & 0 & $5(6.25)$ \\
\hline All-cause mortality, $n(\%)$ at median 19 months & $4(7.1)$ & $17(21.3)-6$ NSTEMI, 11 STEMI \\
\hline In-stent restenosis, $n(\%)$ & $3(5.4)$ & $5(6.25)$ \\
\hline Documented formal/informal MDT, $n(\%)$ & $46(82.1)$ & $44(55)$ \\
\hline
\end{tabular}

Abstract 65 Table 2 Procedural characteristics

\begin{tabular}{|c|c|c|}
\hline & Elective & Acute \\
\hline \multicolumn{3}{|l|}{ Disease anatomy* } \\
\hline - Ostial LMS $n(\%)$ & $11(19.6)$ & $14(17.5)$ \\
\hline - LMS body $n(\%)$ & $-15(26.8)$ & $17(21.3)$ \\
\hline - Bifurcation $n(\%)$ & $-\quad 36(64.3)$ & $56(70)$ \\
\hline - Prox LAD/CXn (\%) & $14(25)$ & $-20(25)$ \\
\hline \multicolumn{3}{|l|}{ PCI technique, $n(\%)$} \\
\hline - Single stent & $47(83.9)$ & $53(66.3)$ \\
\hline - Two-stent & $-9(16.1)$ & $-\quad 27(33.7)$ \\
\hline Optimisation with intracoronary imaging, $n$ (\%) & $42(75)$ & $36(45)$ \\
\hline
\end{tabular}

